\title{
Do Programmers Work at Night or During the Weekend?
}

\author{
Maëlick Claes \\ M3S, ITEE, University of Oulu, Finland \\ maelick.claes@oulu.fi \\ Miikka Kuutila \\ M3S, ITEE, University of Oulu, Finland \\ miikka.kuutila@oulu.fi
}

\author{
Mika V. Mäntylä \\ M3S, ITEE, University of Oulu, Finland \\ mika.mantyla@oulu.fi \\ Bram Adams \\ MCIS, Polytechnique Montreal, Canada \\ bram.adams@polymtl.ca
}

\begin{abstract}
Abnormal working hours can reduce work health, general wellbeing, and productivity, independent from a profession. To inform future approaches for automatic stress and overload detection, this paper establishes empirically collected measures of the work patterns of software engineers. To this aim, we perform the first largescale study of software engineers' working hours by investigating the time stamps of commit activities of 86 large open source software projects, both containing hired and volunteer developers. We find that two thirds of software engineers mainly follow typical office hours, empirically established to be from $10 \mathrm{~h}$ to $18 \mathrm{~h}$, and do not usually work during nights and weekends. Large variations between projects and individuals exist. Surprisingly, we found no support that project maturation would decrease abnormal working hours. In the Firefox case study, we found that hired developers work more during office hours while seniority, either in terms of number of commits or job status, did not impact working hours. We conclude that the use of working hours or timestamps of work products for stress detection requires establishing baselines at the level of individuals.
\end{abstract}

\section{ACM Reference Format:}

Maëlick Claes, Mika V. Mäntylä, Miikka Kuutila, and Bram Adams. 2018. Do Programmers Work at Night or During the Weekend? In Proceedings of ICSE '18: 40th International Conference on Software Engineering, Gothenburg, Sweden, May 27-fune 3, 2018 (ICSE '18), 11 pages.

https://doi.org/10.1145/3180155.3180193

\section{INTRODUCTION}

Poor working patterns can reduce individual health, well-being and productivity. Long working hours have been associated with depression, anxiety, sleep deprivation, and coronary heart disease [1]. A survey study of 35,000 people showed that atypical working hours increased health complaints and poor work life balance even after controlling for the effect of night and shift work [2]. Similar results are found in other papers that highlight health problems related to food digestion and sleeping [3] as well as social impairment with respect to family life [4].

Health problems of poor working patterns are perhaps caused by violation of our natural 24-hour (circadian) rhythm, which has seen

ICSE '18, May 27-fune 3, 2018, Gothenburg, Sweden

(C) 2018 Copyright held by the owner/author(s). Publication rights licensed to Association for Computing Machinery.

This is the author's version of the work. It is posted here for your personal use. Not for redistribution. The definitive Version of Record was published in Proceedings of ICSE '18: 40th International Conference on Software Engineering , May 27-7une 3, 2018, https://doi.org/10.1145/3180155.3180193. a surge of research interest in medicine. Most notably, the research establishing the genetic origins of circadian rhythm was awarded the 2017 Nobel prize in medicine [5]. Recent findings suggest that disturbances in circadian rhythm are the cause (not the effect) of depression and anxiety [6], and that artificial light at night increases the risk of obesity and cancer [7].

Fortunately, weekend recovery has been shown to improve weekly job performance, personal initiative, organizational citizenship behavior, and to lead to a lower perceived effort [8]. Psychological detachment during off-work time reduces emotional exhaustion caused by high job demands and helps to maintain work engagement [9]. Although worker autonomy seems to reduce the negative health and well-being effects of atypical working hours, it does not completely balance them [10]. Other studies of flexible working hours show that they provide affective and work-life benefits [11], which is compatible with the widely accepted results that worker autonomy increases worker well-being stemming (cf. Karasek's job demands control model) [12]. Finally, extensive use of flexible working time is linked with reduced worker productivity [11].

In the software engineering domain, it has been observed that the bugginess of commits, i.e., negative effects on overall developer productivity, is related to the hour of the day (the so-called "circadian work pattern") and to day of the week those commits have been made [13]. However, the results between projects seem to vary. Eyolfson et al. $[14,15]$ propose, based on three well known open source projects (Linux kernel, PostgreSQL, and Xorg), that commits made between 00:00 and 04:00 contain more bugs, while commits made between 07:00 and 12:00 (noon) contain the least. Prechelt and Pepper [16] demonstrate, using data from a closed source industry project, that the most defect-prone hour was 20:00. A hypothesis that unifies these two findings could be that all of those buggy hours might in fact demonstrate the end of a working stint where a developer just wants to be done with the task, leading to prematurely committing the code. Finally, industrial software developers have also developed an interest to night work:

- Quora question with over 100 answers and over 500,000 views on why developers love night work [17]

- entire book with 2,500 paid readers on the topic, written by an industrial programmer [18]

- Stackoverflow analysis with 3,800 Facebook shares and 91 comments of day-night differences [19]

As abnormal working hours can affect occupational health, wellbeing, productivity and staff turnover among software engineers, this paper aims to investigate the work patterns of software developers in large open source projects, either projects with many 
hired resources (Mozilla) and those without (Apache Foundation), as well as in a (local) Finnish IT company. We are particularly interested in the degree to which work is performed outside of the commonly expected working hours, since, based on the literature discussed above, such irregular working hours may act as a proxy for job-related stress and time pressure conditions and suggest non-sufficient detachment from work.

The Mozilla Foundation is known for Firefox, which makes an interesting case study as the core software engineers are paid to develop it. In addition, Mozilla also hosts projects that have been gradually abandoned and left to the community, such as Thunderbird and SeaMonkey. The Apache Foundation is an organization supporting open source projects and their communities of developers, but does not pay its developers. Some of its projects are business-critical to companies, who pay developers to work on them, while others consist of hobbyist developers. Finally, the local company provides a reference to compare the groups of open source projects to, since a closed source company follows a more traditional work schedule and larger financial concerns are at stake.

First, we investigate the following research questions for all considered projects:

\section{RQ1 What are the circadian and weekly work patterns of software developers?}

RQ2 How does the usual work pattern vary across different projects?

RQ3 Are office hour commits different in terms of size?

RQ4 Is there a difference in the developers' work patterns over time?

Then, because results of RQ5 can considerably vary due to differences in terminology between projects, we focus solely on Mozilla Firefox. Moreover, the identification of developers background needed for RQ6 involves a lot of manual work and is too laborious to be performed for all projects. Furthermore, Firefox is the largest project of our data set as it contains 228,697 commits, which represents roughly one third of all of the commits of our data set. Finally, Firefox includes both paid work and voluntary contributions.

RQ5 Are office hour commits different in terms of content? RQ6 Can demographics explain office hour activity?

This paper is structured as follows. We discuss related work in Section 2. In Section 3, we give details on our data extraction process, then we address our research questions in Section 4 and Section 5 . We then present the threats to validity that can impact our results in Section 6 and conclude in Section 7.

\section{RELATED WORK}

Sall et al. [20] studied weekend work activity patterns in the San Francisco Bay Area using surveys. Their results indicate that a host of variables affect the likeliness of working during the weekend, in particular, gender, race, type of work and income. Individuals are more likely to work out of home during weekends in the winter season than in other seasons.

Wang et al. [21] examined work patterns of scientists by looking at the amount of scientific papers being downloaded on different days. Scientists work $60-70 \%$ as much of their time during the weekend as during the week. Time worked during weekends differs by country: scientists work proportionally more during weekends in China than in the USA and Germany.

Binnewies et al. [8] investigated the importance of recovery during weekends and its implications on work performance. Data from surveys indicate that experiencing psychological detachment, relaxation and mastery during weekends was positively correlated with being recovered at the beginning of the working week, which in turn was positively related to self-reported work performance.

McKee [22] investigated the reasons for increased mortality rate in hospitals during the weekend, with explanations ranging from more seriously ill patients to less experienced staff. In extreme cases, the weekend effect to lead to $44 \%$ higher odds of mortality on Friday compared to Monday [23]. However, multiple sources state conflicting evidence on the source of this effect [22-24].

Some relevant studies also exist in software engineering. Industrial blogs on GitHub [25] and StackOverflow [19] report that less main stream languages such as Haskell are more commonly used during the night than languages adopted in the industry such as Java. Multiple studies [26-28] proposed multi-objective techniques to support project planning avoiding overtime.

Khomh et al. [29] studied the impact of Firefox's fast release cycle on post-release bugs and found that not only did the new release cycle not increase the number of bugs, bugs were also fixed faster. Although most of the studies thus far on the switch of release cycle have focused on its quality assurance implications, the repercussions for developers in terms of work quality have largely been ignored.

Our previous work [30] studied abnormal working hours on two projects only, while this paper performs a large-scale study on 86 open source and 1 industrial project. Furthermore, we use more advanced methods: k-means clustering and a dynamic search of office hours instead of a static heuristic.

To summarize, although some initial evidence has been found regarding the interplay between work and well-being in software engineering, a structured analysis of (un)healthy work patterns is missing. This paper starts to fill this gap by studying and comparing the periods during which developers are actively working in a software project.

\section{DATA EXTRACTION}

We mined development data from the Git and Mercurial repositories of Mozilla ${ }^{1}$ and Apache ${ }^{2}$ and of a local company's product. The local company's product contained more than 20,000 commits from nine developers. We have visited the company several times to ensure the validity and usefulness of our work. We then wrote custom scripts to extract the list of commits (code changes), associated timestamps and authors from all code repositories.

For Mozilla, we needed to do extra processing in order to find out which commit belongs to which project. First, we used the GrimoireLab tools ${ }^{3}$ to extract issue comments from Mozilla's Bugzilla repository ${ }^{4}$ (i.e., the database containing reported issues, such as bug reports or feature requests). Second, we linked commit messages to the corresponding issue report by looking for an issue

\footnotetext{
${ }^{1}$ https://hg.mozilla.org

${ }^{2}$ https://git.apache.org/

${ }^{3}$ https://grimoirelab.github.io/

${ }^{4} \mathrm{http}: / /$ bugzilla.mozilla.com
} 
identifier in a given message. Out of the 396,180 extracted commits, 330,078 were successfully linked to a bug issue. After linking, we then filtered the commits to only keep the ones related to the following major products: Firefox, Core, Firefox OS, Firefox for Android, Thunderbird and SeaMonkey.

While the history of each Apache project is stored in an individual git repository, we realized that many of these old commits were missing the time zone information. These commits might have been imported from another version control system that did not store such information. Thus, for each project, we only considered commits starting from the first commit with a timezone different than UTC (i.e., the first commit that is clearly made after the import from the old system). Moreover, we only considered Apache projects with at least 2,000 commits after filtering, which reduced the number of Apache projects from 822 to 81 . This left us with a total of 451,116 commits.

In order to study the work patterns of individual developers, we performed a basic merging of the different authors' identities. We first cleaned the name and email used in the version control system's author field. Then we grouped together identities using the same name or email addresses. Finally, two of the authors manually checked the result in order to avoid any false positive.

\section{EMPIRICAL ANALYSIS OF THE WORK PATTERNS OF ALL PROJECTS}

\section{RQ1. What are the circadian and weekly work patterns of software developers?}

Motivation. Given the well-being and health risks related to abnormal working hours, we wish to establish empirical baselines that later may be turned into normative guidelines to ensure the well-being and health of software developers.

Commits follow a weekly rhythm. Fig. 1a, 1c and 1e show the number of commits made on each day of the week within the Mozilla (319,139 considered commits), Apache (574,563 commits) and the local company projects (22,193 commits), respectively. We can clearly see that fewer commits are being posted during the weekend, while there is a slight variation in activity during the week, with Monday and Friday being the least active days in all cases. Tuesday is the most active day for the Mozilla and Apache projects, while in the local company it is Thursday.

Commits follow a circadian (24-hour) rhythm. The circadian rhythm is present in Apache, Mozilla and in the local company projects, see Fig. 1b, 1d and 1f. The lowest activity for all projects happens during the night, with the number of commits starting to increase in the morning until a dip in activity happens during lunch hour. In the Mozilla and Apache projects, lunch mostly happens at 12 o'clock, while in the local company it happens at 11 o'clock (something which we confirmed through face-to-face discussions with the company).

After lunch hour, the number of commits increases again until a decreasing trend in commits starts setting in from $4 \mathrm{pm}$ in all three data sets. For the local company, we can also see an unexpected dip in commits at $2 \mathrm{pm}$, yet the company was not able to explain this phenomenon to us. The decreasing trend in the number of commits plateaus at $7 \mathrm{pm}$ for the Apache and Mozilla projects, while for the local company, we can see that work during the evenings is very limited compared with the Mozilla and Apache projects.

Working hours of software developers are typically from 10-18. If we consider a 40 hour work week, which is still the norm for most professions, each week day should correspond to about 8 working hours. Hence, in order to determine the working period for each of our projects, we searched the week days for the eight-hour stint that covers the largest share of commits in a day. First, for a given project, we computed all the $(\mathrm{HH}, \mathrm{mm})$ tuples representing a timestamp (in 24 hours format) for which at least one commit was made during a weekday. For each $(\mathrm{HH}, \mathrm{mm})$ tuple, we computed the number of commits made during the 8-hour interval $\left[(H H, m m),\left(H_{+8}, m m\right)[\right.$. We then selected the interval with the highest number of commits as the 8-hour office period of the considered project.

The results of this search are shown in Table 1. For 34\% (30/87) of the projects, this search results in working hours starting between 09:00 and 09:59, and for 45\% (39/87) of the projects, the working hours start between 10:00 and 10:59. The median start time of work across all projects is at 10:03.

$\mathbf{6 6 \%}$ of developers follow office hours. We were interested in seeing if we can find different work patterns across the weekly cycle and the daily circadian rhythm of different developers within our data set of 87 software projects, and how those patterns look like. While past work has considered the core contributors to be the top $20 \%$ of the committers [31], in our data set it only takes 22 commits total to be in the top, which we think is not enough for establishing weekly working patterns. Thus we only consider the top $10 \%$ of developers, leaving us with 1,108 developers (out of $11,059)$, each with 95 or more commits. This top $10 \%$ of developers has made $88 \%$ out of all commits of our data set.

For clustering purposes, we computed the relative share of commits made by each hour of the week by each developer. Thus, for each developer, we end up with a feature vector of 168 elements, one for each hour of the week. Similar to previous work on weekly patterns of mobile phone usage behavior [32], we then used kmeans clustering on the vectors. We tested values of $\mathrm{k}$ (i.e., the number of clusters) from 2 to 7 . We observed that going beyond three clusters did not bring new information, instead clusters with very few individuals and high noise started to appear. Fig. 2 shows the weekly work patterns (the feature vectors of centroids) of each of the three clusters.

The figure shows that two clusters (green and black lines) follow typical office hours where commits are concentrated on day time hours during the work week, with clear dips during lunch hour. The green cluster starts and stops working a bit earlier than the black cluster. In fact, when we shift the green cluster by one hour, we can see in Figure 2 that both clusters have highly similar work patterns. These two clusters contain $66 \%$ of all developers, and they roughly correspond to the projects starting at 9am vs. 10am in Table 1.

However, Fig. 2 also shows a third cluster (blue) that does not follow the regular office hour pattern. Developers in this blue cluster commit mostly from noon until midnight during the weekdays and weekends. The difference between the blue cluster and the other two clusters is that this group works more during evenings and weekends than the other two clusters. 




(a) Daily for all Mozilla projects

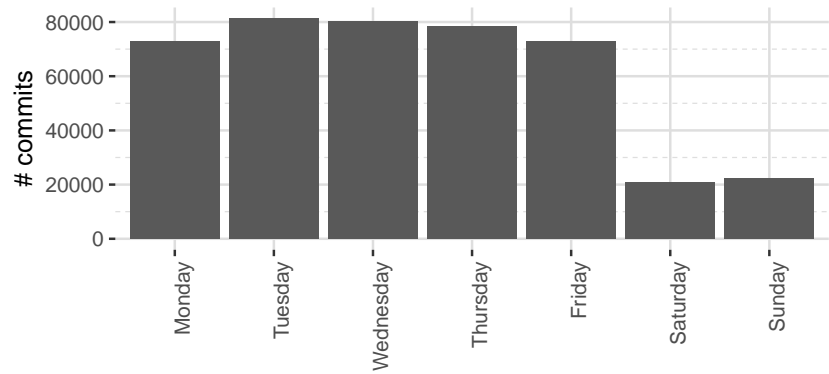

(c) Daily for all Apache projects

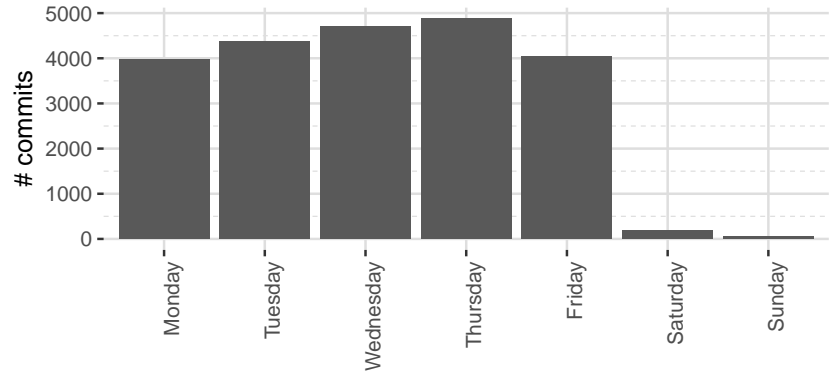

(e) Daily for the local company

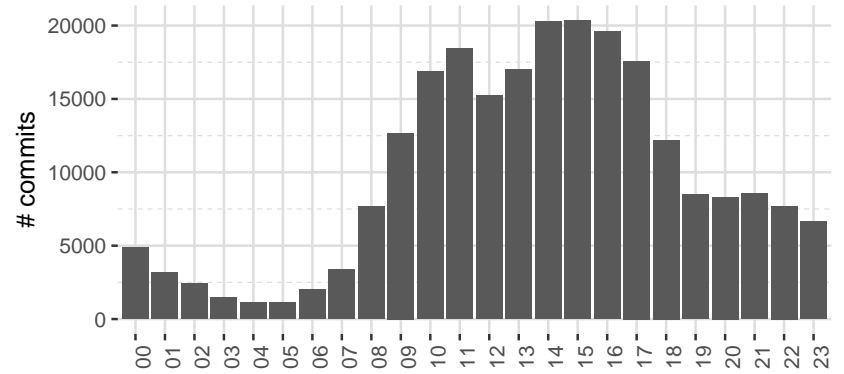

(b) Hourly for all Mozilla projects

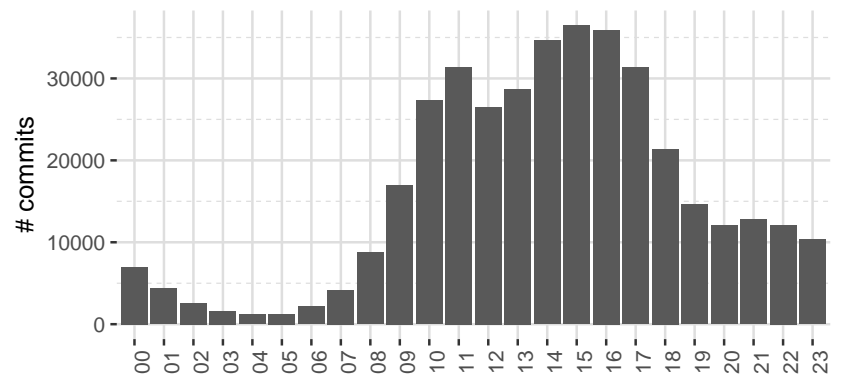

(d) Hourly for all Apache projects

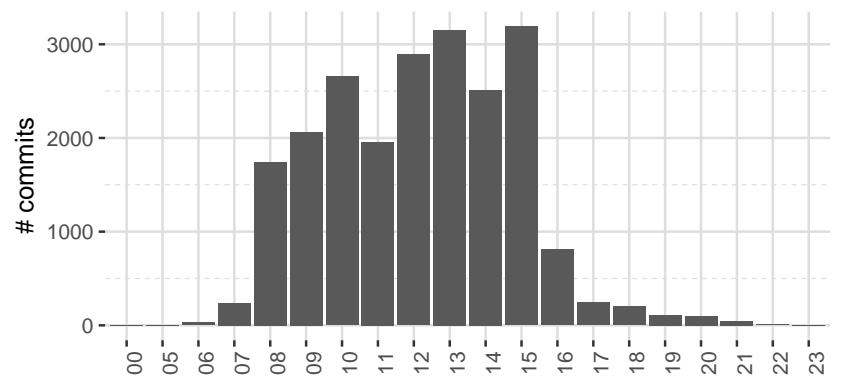

(f) Hourly for the local company

Figure 1: Distribution of the number of commits across the days in a week and the different hours in a weekday.

Table 1: Start of working hours for projects in our data set.

\begin{tabular}{l|l|l|l|l|l|l|l|l|} 
Start time & $08: 00-59$ & $09: 00-59$ & $10: 00-59$ & $11: 00-59$ & $12: 00-59$ & $14: 00-59$ & $16: 00-59$ & $17: 00-59$ \\
\hline \# Projects & 4 & 30 & 39 & 8 & 2 & 1 & 2 & 1
\end{tabular}

Discussion: Up until now, we have established that a weekly and circadian rhythm is followed in the projects of Apache and Mozilla foundation, as well in the local company product that was used as a comparison. We have also established a way to determine the eight-hour work day start and end times for each project, then presented measurements of how much work gets done during the typical working hours. Establishing the normal working hours is important (especially in a distributed context) if we wish to determine whether the developers of a particular project are, for example, under time pressure and forced to work overtime.
Committing $70 \%$ of work during typical working hours might be a stress signal for a project that has previously had a share of $90 \%$, but not for another project that constantly commits $70 \%$ work during typical working hours. We also found that two thirds of the developers are clustered to groups that follow office hours. In the two office hour groups, we saw a phase shift of a bit more than one hour. We think that this phase shift is due to both project culture and individual preferences, as some individuals are known as morning persons while others are not [33]. This suggests that clustering of individual developers may allow personalized stress 


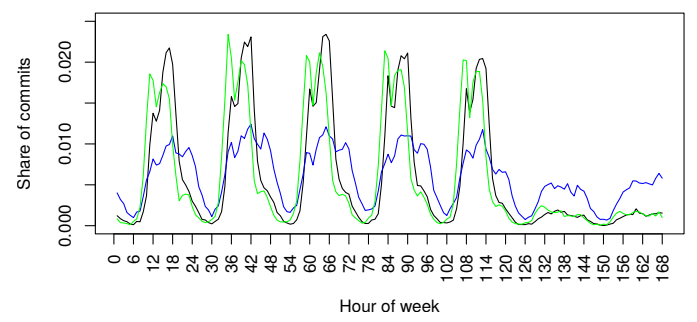

Figure 2: Weekly work patterns for the three k-means centroids corresponding to the 1,108 top software developers.

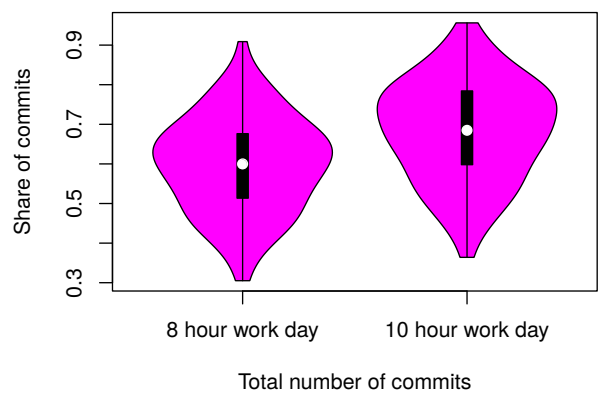

Figure 3: Distribution of percentage of commits across projects in an eight-hour typical work day and ten-hour extended work day (eight-hour day plus/minus one) in our data set of 87 projects.

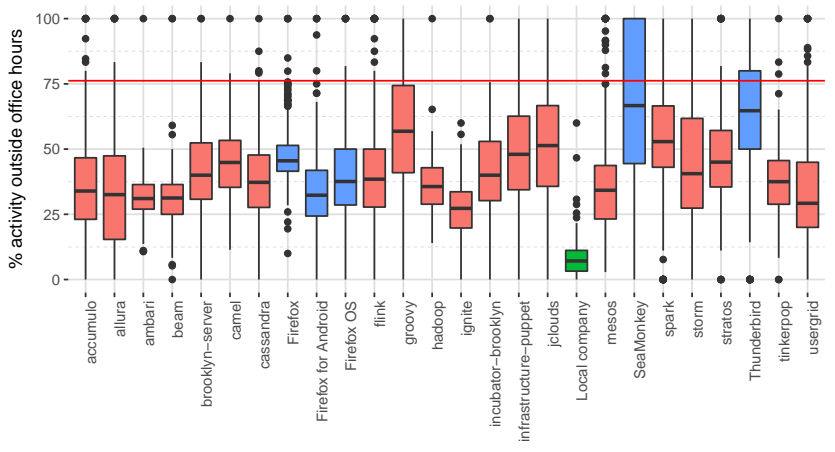

Figure 4: Variation within the local company project (green), the Mozilla projects (blue) and the top-20 Apache projects (red) of the distribution across commits done outside typical working hours per week.

or overtime detection, for at least two thirds of the developers that follow regular rhythm.

\section{RQ2. How does the usual work pattern vary across different projects?}

Motivation: Here, we investigate variations in working hours between projects. After all, work patterns are shaped by both individual preferences and project culture and norms.

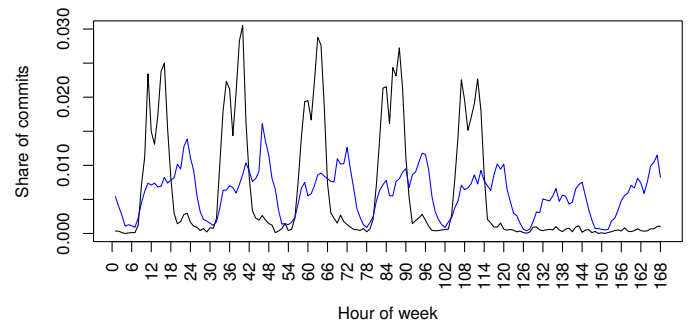

Figure 5: Two extreme clusters with respect to working hours with five (Black) and six (Blue) projects respectively.

$\mathbf{9 0 \%}$ of the projects have similar typical working hours. This can be observed from Table 1, which shows how 78 out of 87 projects work from 10-18 plus/minus one hour. Variations in work patterns between projects are still considerable as the amount of commits done in eight-hour work days is $60 \%$ on average with a standard deviation of $12.0 \%$ (min-max $31-91 \%)$. Moreover, the extended typical hours ( 8 hours plus/minus one hour) has an average of $68 \%$ with a standard deviation of $12.6 \%$ (mix-max: 36$96 \%$ ). On average, only $60 \%$ of work gets done during typical working hours. The violin plot in Fig. 3 shows the distribution across projects of the percentage of commits performed during working hours.

Figure 4 shows the weekly variation within the most active projects. The differences between projects in terms of relative activity happening during 8-hour work days can partially be explained by the presence of paid developers. In particular, the three projects with the most activity outside office hours (SeaMonkey, Thunderbird and Groovy) are projects that are now community projects.

The amount of activity outside office hours during the week and during the weekend are correlated. The percentage of activity in a project outside office hours during weekdays, and the activity during weekends yield a correlation coefficient of 0.77 (pearson), with a p-value of $<0.001$. When considering developers (with at least 100 commits) instead of projects, we obtain a correlation coefficient of 0.62 . This means that both projects and developers that work a lot outside office hours are also likely to work a lot during the weekend.

Clustering allows the separation of projects based on work patterns. We again used k-means clustering (with $k=7$ ) by the hour of the week. The difference is that here we cluster by project $(n=87)$ while previously we clustered the top $10 \%$ individuals $(n=1,108)$. Fig. 5 shows the two most extreme clusters: the black one follows office hours rigorously, while the blue one has a commit peak right before midnight. In the rigorous office hour group (5 projects) we have our local company but also open source projects like Apache Cordova for Android and Apache Geode. The other group (6 projects) contains projects like SeaMonkey and Thunderbird, which are community projects (i.e., very little paid work from the Mozilla foundation) and also the logging library $\log 4 \mathrm{j} 2$. The other clusters, not shown in the figure, fall between these two extreme clusters.

Discussion: Important differences between projects can be observed in terms of activity outside regular office hours. Although no open source project follows office hours as well as the local Finnish 
company, some open source projects follow them more closely than others. In particular, community projects such as SeaMonkey, Thunderbird and Groovy tend to contain a lot of activity outside office hours.

\section{RQ3. Are office hour commits different in terms of size?}

Motivation: Given that developers are more active during office hours than during the other hours of the week, we wanted to check whether the commits made during office hours are different in terms of size and content in comparison to outside-office hour comments. Previous work has shown that different programming languages are used during the night $[19,25]$ and that night work is more technical [30]. In this paper, we approximate the dichotomy of office hours versus non-office hours using our dynamic office hour detection approach, which ensures that, for each project, only the most active weekday hours are taken into account as office time.

We found no significant difference in terms of added lines of code, with a Mann-Whitney U test p-value of 0.076, Cliff's delta $<0.00$. Since our projects are of different sizes, and since a large project like Firefox may mask effects present in smaller projects, we checked each project individually and found that for $16 \%$ of them, there is no difference in the median commit size. For $33 \%$ (29/87), the office hour commits are larger but only for $11 \%(10 / 87)$ this difference is statistically significant with Mann-Whitney U test alpha level 0.05 . On the other hand, for $51 \%$ (44/87) of the projects, the office hour commits are smaller, while only for $26 \%$ (23/87) of the projects a statistically significant difference was obtained. We checked the Cliffs' Delta effect sizes for all projects and they ranged from 0.10 to -0.11 , meaning a negligible effect in all cases [34].

For lines of code removed, we found a significant difference (Mann-Whitney $U$ test $\mathbf{p}$-value $<0.001$ ), but the effect size is negligible (Cliff's delta $<0.00$ ). Checking each project individually shows that for $26 \%$ (23/87) the office hour removals are larger (8\% (7/87) significant), for $44 \%(38 / 87)$ they are smaller (21\% (18/87) significant), and for $30 \%(26 / 87)$ of the projects there is no difference in the median number of lines removed. The effect sizes were again negligible ranging from 0.08 to -0.10 .

Discussion: In terms of commit size, we conclude that there is no difference in practice between office hours and outside office hours.

\section{RQ4. Is there a difference in the developers' work patterns over time?}

Motivation: While a software project could start off on schedule, with most of the work happening during office hours, deadlines and delays are notorious for introducing outside office work or even death marches. On the other hand, we could hypothesize that a project may initially have a start-up culture with highly flexible working hours, while later, as the project matures, more work would start to happen during office hours. We are interested which of these conflicting ideas, i.e., 1) starting on time with eventual delays ending up in death march versus 2) starting with a startup culture and maturing as time passes, is more prevalent in our projects.

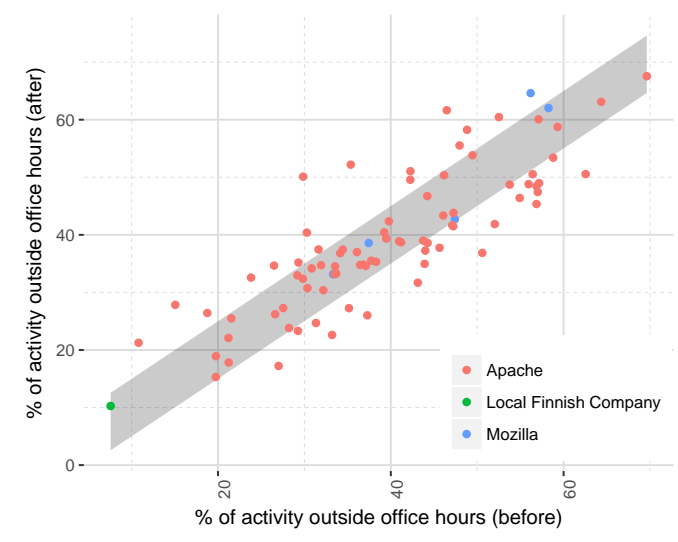

Figure 6: Percentage of activity outside office hours during the first and second half of each project's analyzed time period. Projects in the gray area did not experience an increase or decrease of activity of more than $5 \%$ outside office hours.

For 18 projects, the activity outside office hours increased by at least $5 \%$, while for 27 projects it decreased by at least $\mathbf{5 \%}$. For each project, we split the activity history in two equal parts (in terms of number of commits) in order to find whether the work pattern changed over time or not. Fig. 6 shows that for 46 projects the activity outside office hours activity neither increased or decreased by more than $5 \%$.

Examples of Apache projects where activity outside office hours increased the most are Allura, from $26.5 \%$ to $34.7 \%$, Groovy from 48.8 to $58.2 \%$, and Wicket from 29.8 to $50.1 \%$. Apache projects that saw their activity drop include Drill from 37 to $24.4 \%$, AsterixDB from $57 \%$ to $47.4 \%$, Spark from 54.9 to $46.4 \%$ and Cassandra from 43.1 to $31.7 \%$. For Mozilla, SeaMonkey experienced a significantly increased activity outside office hours, from $56.2 \%$ to $64.6 \%$ and Thunderbird from $58.2 \%$ to $62 \%$. For Mozilla, only Firefox experienced a significant decrease from $47.4 \%$ to $42.7 \%$.

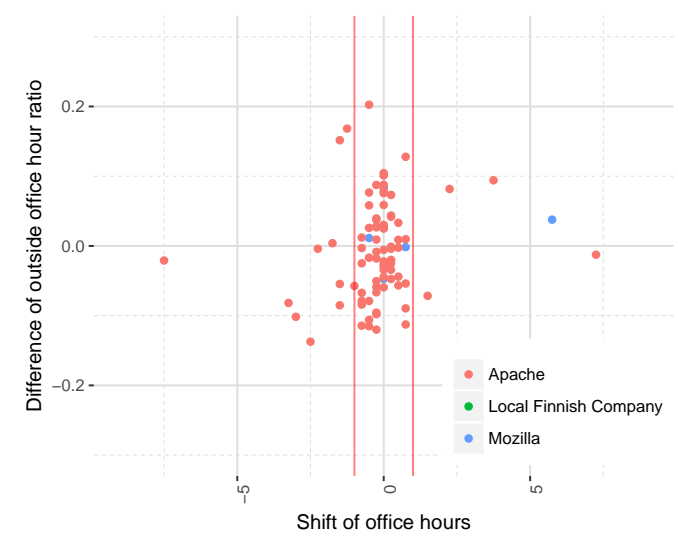

Figure 7: Differences of relative activity outside office hours over time ( $\mathrm{Y}$ axis), as observed in Fig. 6, and shifts of office hour period over time ( $\mathrm{X}$ axis). 


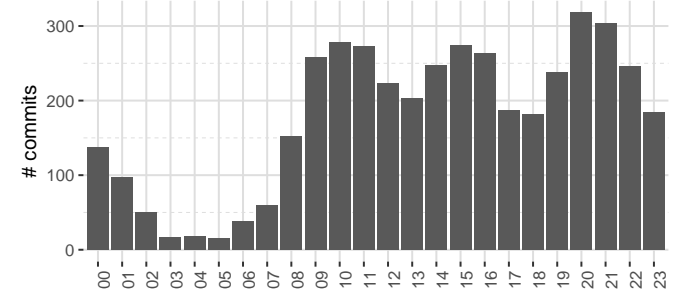

Figure 8: Distribution of the number of hourly weekday commits for Thunderbird.

In Fig. 6, we identified separate office hours for each half of the projects' history, since the eight-hour office hour time interval can change over time. As shown in Fig. 7, most projects do not encounter a major shift of their time interval. Indeed, most projects (75/91) experienced a shift of less than one hour (X axis). Only 5 projects experienced a positive shift (towards the evening) of more than one hour, and 11 a negative shift (towards the morning) of more than one hour.

A significant negative shift usually means that the identified 8hour period shifts shift towards common office hours. For example, Apache Log4j shifted from an 8-hour period starting at 17:15 to one starting at 09:45. On the other hand, a positive shift usually means that the 8-hour period moves away from common office hours. For example, Mozilla Thunderbird experienced a shift from 08:45 to 14:30. However, in both of these cases the amount of work done outside these 8-hour periods didn't change a lot and is relatively high (from 0.58 to 0.62 for Thunderbird and from 0.69 to 0.68 for $\log 4 \mathrm{j})$.

Overall, we did not find any correlation between the amount of office hour work and shift of office hours period. Most of the projects that experience large shifts have a rather high amount of activity outside the identified office hour period. One possible explanation is that commit activity is more spread throughout the day for these projects, making the identification of an 8-hour stint more sensitive to small variations. For example, as seen in Fig. 8, Thunderbird has a high level of activity from 8 am to 1 am with activity peaks around $10 \mathrm{am}, 3 \mathrm{pm}$ and $8 \mathrm{pm}$.

Discussion: In summary, most projects do not experience large changes over time in their work pattern. Important changes in the identified 8-hour office hour period are often found in projects with commits being more spread throughout the day.

\section{DEEPER ANALYSIS OF THE WORK PATTERNS OF MOZILLA FIREFOX}

\section{RQ5. Are office hour commits different in terms of content?}

Motivation: This question is similar to RQ3, which looked for differences between the size of commits within and outside office hours, however here we investigate the content of the commits, by comparing word clouds. Figure 9 shows a comparison word cloud of commit messages for Mozilla Firefox, which has roughly 230,000 commits. As the visualization of a comparison cloud tends to make
Table 2: Commit message differences (in \%) during vs. outside office hours.

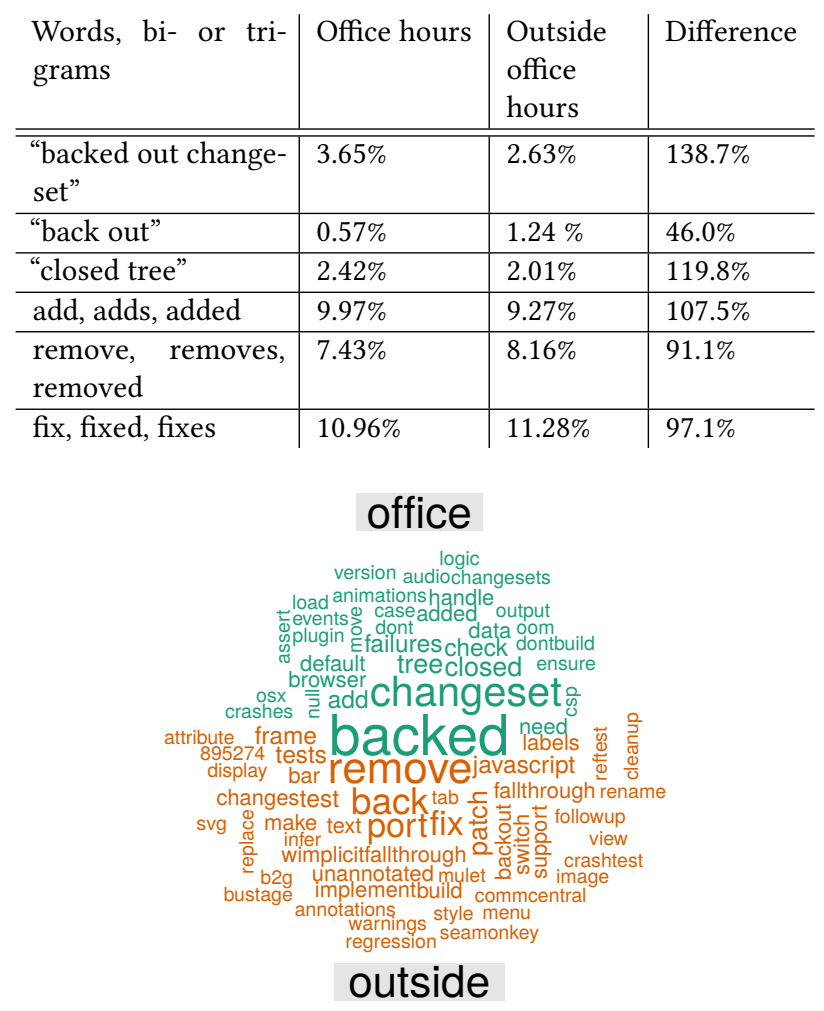

Figure 9: Comparison of the clouds of words used during and outside office hours.

the differences appear larger than they actually are, Table 2 also shows percentage differences for the selected terms.

Developers back out more code themselves outside office hours. During office hours, there are more commit messages containing the "Backed out changeset" statement, which is part of the official terminology of the version control system used to indicate that a commit (change set) has been reverted (after being merged). Furthermore, "Backed out changeset $<$ change set id $>$ " is often followed by the explanation such as "because of a possible Talos regression" 5 . For outside office hours there are more commit messages with "Back out" (instead of "Backed out changeset"), this informally seems to indicate that, after office hours, a developer is backing out changes herself rather than being backed out by some authority.

A "Closed tree" message in a commit is used to indicate that a commit also closes a version control tree. together with a "Backed out changeset" message. In fact, the "closed tree" message is proportionally more common outside office hours when we remove the commits where it appears together with the "Backed out changeset" message ( $1.30 \%$ vs $1.41 \%)$. Table 2 also shows that work involving adding something is more common during office hours while removals and fixes are more common outside office hours.

\footnotetext{
${ }^{5}$ Talos is the performance testing framework used by Mozilla.
} 


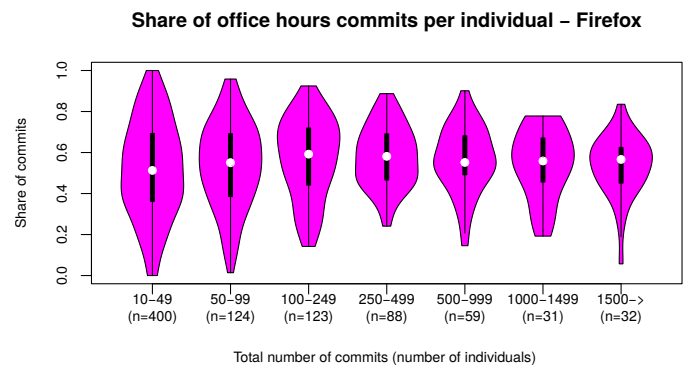

Figure 10: Share of office hour commits for individuals, for various numbers of total commits.

Discussion: We found small differences between office hour and outside-office hour commit messages. The most notable difference was that during office hours there are more formal reverts made to version control. Outside office hours, informal reverts made by the developer herself are more common.

\section{RQ6. Can demographics explain office hour activity?}

Motivation: First, we hypothesize that more senior developers will likely work more during office hours, as they are more likely to be paid for their efforts. For this purpose, we investigated whether there was a correlation between the number of commits made by a developer and the amount of abnormal work. We only considered individuals with 10 or more commits, which reduces the number of analyzed individuals in Firefox significantly from 2,755 to 857, but still retains $98 \%$ of the commits.

Second, one explanation for work outside-office hours in open source projects is the amount of paid and non-paid contributors. If developers are hired by a company to work on a project, they are more likely to work during regular office hours. On the contrary, if they are not paid, they are more likely to work during their free time. Hence, we ran a manual background check for the top $10 \%$ of developers to determine whether they are paid or non-paid, leaving us with 278 developers (out of 2,755), each with 147 or more commits. This top $10 \%$ of developers has made $87 \%$ out of all the Firefox commits. Our background check considered information available online on websites such as LinkedIn or the developers' personal websites (e.g., publicly available CVs). In addition to their job status, we also gathered information about their location, experience in the software industry and position at Mozilla.

We did not find a statistically significant correlation between developer seniority and amount of office hour work. Fig. 10 divides the contributors into buckets based on their number of commits. The violin plots show that the amount of office hour work varies between groups. We can see an increase in the amount of commits during office hours as the number of commits increase in the first three groups. However, this increase plateaus at the 4th cluster of developers (between 250-499 commits). Then, for the top contributor group with 1,500 or more commits, the share of abnormal commits increases again. Overall, it seems that the number of commits is not a very good predictor of office hours work.



Figure 11: Clusters of work patterns for the top $10 \%$ Firefox developers.

Projects were clustered within 3 work pattern clusters, two of which mostly used office hours Fig. 11 shows the clustering of three different work patterns that were identified using k-means clustering. Since we are interested in the differences between office hour commits and outside-office hour commits within a single project, we used our dynamic office hour setting data for Mozilla Firefox and divided each week into 12 time units. From Monday through Friday, a commit can happen either during office hours (normal) or outside-office hours (abnormal), yielding 10 time units. For weekends, we consider both Saturday and Sunday as abnormal, which gives two more time units. Reducing the time units in a week (X-axis) from 168 hours to 12 periods also makes the data less noisy and gives a visualization that is easier to comprehend. Similar to Fig. 2, Fig. 3, and Fig. 5, the Y-axis in Fig. 11 represents the share of commits done in a particular time unit.

In Fig. 11, we can see one group in cluster $(n=101)$ that mostly commits during office hours. This office hour cluster has the highest share of commits (15.9\%) on Tuesday during office hours and the lowest on Sunday (1.7\%). On the other hand, the extreme (black) cluster $(n=46)$ works more during abnormal times, and has the highest share of commits on Saturday (11.4\%) and the lowest on Monday during office hours (5.7\%). The third cluster in blue $(n=131)$ lies between these two extreme clusters and obtains the highest share of commits on Wednesday during office hours (11.2\%) and the lowest on Sunday (3.9\%). This group substantially works outside office hours during the week, but less during weekends. For weekends, one needs to consider that a single data point represents all the commits for the day, while for a weekday the commits are divided into office hours and abnormal commits.

For the three clusters in Fig. 11, we ran the dynamic office hour heuristic and found that the green and blue clusters both have office hours from 10:00-18:00. During those hours they perform 72 and $53 \%$ of their weekly commits, respectively. The third cluster commits $34 \%$ during their office hours, which are from 15:15 to 23:15, indicating a very irregular and abnormal working pattern. Office hours for the entire Firefox project were 10:00-18:00, and during that time the entire project completed $55 \%$ of their commits. Thus, the middle group represents the project average while the other two groups show extreme variations.

Outside office hour work is mostly performed by unpaid developers. We compared the clusters identified in Fig. 11 with our manually extracted information about Mozilla paid developers. Table 3 shows the absolute number and relative percentage of paid developers in each cluster (first two numbers in each cell). While $90 \%$ of the developers in the cluster with the largest amount of 
Table 3: Clustering of (un)paid top $10 \%$ developers of Firefox. The first number and first percentage respectively indicate the number and percentage of developers in a given cluster that is (un)paid, while the second percentage indicates the percentage of (un)paid developers that are within a cluster.

\begin{tabular}{l|l|l|l} 
Paid & $\begin{array}{l}\text { Outside office } \\
\text { hours cluster }\end{array}$ & Average cluster & $\begin{array}{l}\text { Office hours clus- } \\
\text { ter }\end{array}$ \\
\hline No & $23(50 \% / 47.9 \%)$ & $15(11.6 \% / 31.3 \%)$ & $10(10 \% / 20.8 \%)$ \\
Yes & $23(50 \% / 10.1 \%)$ & $\begin{array}{l}114(88.4 \% \quad / \\
50.2 \%)\end{array}$ & $90(90 \% / 39.7 \%)$
\end{tabular}

Table 4: Median percentage of office hour commits depending on location, experience in the software industry and position at Mozilla.

\begin{tabular}{|c|c|c|c|c|}
\hline Characteristic & \# dev. & \# commits & $\begin{array}{l}\text { office } \\
\text { hours } \\
\text { commits }\end{array}$ & $\begin{array}{l}\text { beginning } \\
\text { of office } \\
\text { hours } \\
\text { period }\end{array}$ \\
\hline $\begin{array}{l}\text { Based in Eu- } \\
\text { rope }\end{array}$ & 65 & 41,962 & $54.4 \%$ & $9: 45$ \\
\hline $\begin{array}{l}\text { Based in Amer- } \\
\text { ica }\end{array}$ & 118 & 78,070 & $60.9 \%$ & $10: 00$ \\
\hline $\begin{array}{l}\text { Senior posi- } \\
\text { tion }\end{array}$ & 78 & 47,483 & $57.6 \%$ & $10: 00$ \\
\hline $\begin{array}{l}\text { Non senior po- } \\
\text { sition }\end{array}$ & 197 & 130,468 & $59.5 \%$ & $10: 00$ \\
\hline $\begin{array}{l}\text { Manager po- } \\
\text { sition }\end{array}$ & 19 & 7,957 & $63.1 \%$ & $10: 00$ \\
\hline $\begin{array}{l}\text { Non manager } \\
\text { position }\end{array}$ & 256 & 169,994 & $58.6 \%$ & $9: 30$ \\
\hline
\end{tabular}

office hour commits (last column), and $88 \%$ of the developers in the average cluster (second-to-last column) were paid, only $50 \%$ of the cluster with most outside office hours (second column) were paid by Mozilla. This corresponds to only $10.1 \%$ of all paid developers. In other words, paid developers work less outside office hours than unpaid ones. Yet most paid Firefox developers still work significantly more outside office hours than developers from the local company

There are no major differences in office hour work between developer profiles. Table 4 shows that the developers based in Europe work less during office hours. It seems that career moves on the technical ladder, i.e., having a title with the word "senior" or "principle", decreases the median office hour work by $1.9 \%$. However, career moves on the managerial ladder, i.e., having a title with the word "manager", decrease the outside office hour commits by $4.5 \%$. We suspect that management positions require less technical work and more communication and coordination, which might explain this difference. In other words, for managers, using commits as working hour indicators could be misleading. However, when running a Mann-Whitney U test, only the difference between Europe-based and America-based developers is statistically significant with a p-value $<0.01$.
Discussion: We did not find any differences between the seniority of developers when measured in terms of commits. On the other hand, developers following office hours more closely are usually developers paid by Mozilla, even though most paid developers still work a lot outside regular office hours. We did not observe any strong characteristics of paid developers that could explain these differences. The only statistically significant difference is observed between European and American developers, with the former following office hours less than the latter. A potential explanation is the difference in time zone and the fact that Mozilla is based in North America. Finally, the office hour work seems to be impacted by job title, but again the differences are not statistically significant.

\section{THREATS TO VALIDITY}

Regarding construct validity, commit authoring times used in version control systems might not be representative of the actual time period when the code change was written: a timestamp cannot provide information about the time actually spent working for that commit. However, as we investigate weekly and daily rhythms, which are accumulated over months and years, this should not significantly affect the results. For example, if a developer works at a regular rhythm from nine to five, then, over time, all commits would occur between these regular working hours, with perhaps a small lag from the start of working until the first commit. Furthermore, the fact that in most figures we observed dips in commit count during typical lunch hours strengthens the idea that commit time stamps are reliable when used for daily and weekly working hour discovery.

However, for the local company, we investigated the developers' chat log activity and commit activity. We found that not only are these strongly correlated $(r=0.74)$, but the "start of the day lag" between the 8-hour office period based on the chat log timestamps and the one based on the commit timestamps is only 15 minutes. This offers further support that commit time stamps can be used to study what are the typical working hours in daily and weekly level.

Another threat to construct valididty is that our analysis depends on data extracted from repositories available from online sources. Although we found some missing time zone information for Apache projects and filtered them out, other errors or incompleteness in these data sources may impact the result of our analysis. In particular, we relied on time zone information available from Mozilla's Mercurial source code repository and Apache source code repositories. The time zone from this repository might not be accurate enough to pinpoint the developer's actual position.

In order to identify developers hired by Mozilla, we manually looked for information about them online. Although this allows us to identify a large amount of the most active developers as hired by Mozilla, we might have missed developers who do not share their CV online. We also merged developers' identities using a very basic identity merging technique. We manually checked for false positives in order to avoid merging the work pattern of two developers as a single one and thus overestimating their amount of activity. However, there might be false negatives remaining, which could be particularly problematic in the case of developers using 
their work e-mail during office hours and personal emails outside office hours.

Regarding threats to external validity, our study only includes open source projects from Mozilla and Apache, and one project from a local company. The results obtained are specific to those organizations' culture and to the habits of their developers. Although the results reveal important differences between the projects, as illustrated in Figure 5, we cannot guarantee that our data set would be representative of the entire industry in particular, as we would need more closed source projects.

\section{CONCLUSION AND FUTURE WORK}

In this paper, we have performed a first large-scale study of software developers' work patterns by investigating over 700,000 version control commits coming from 87 software projects, each having a minimum of 2,000 commits. We were motivated to investigate work patterns as according to medical and occupation well-being literature they might be possible indicators of overload, time pressure, and unhealthy working patterns.

We found that developers follow a typical circadian rhythm where most of the work is performed during day time and less work is performed during evenings and at night. Dips in activity during typical lunch hours can also be seen. The weekly rhythm shows that during weekends there is radically less activity. We then established a search method that finds, for each project or individual, the period, of a given length, with the highest number of commits. In this paper, we fixed the length of time series to eight hours to make comparisons between projects. Our method can also be used in reverse to find a continuous time series that results in a certain percentage of commits.

We used our search method to find that the median 8-hour working day for software engineering projects in our data runs from 10:00 to 18:00 instead of the typical 09:00-17:00 (nine-to-five). Thus, the often quoted idea of programmers as night owls that has even made it to a book titled "Why programmers work at night" [18] holds only partially. Perhaps the plus one hour shift compared to regular nine-to-five professions gives the impression of a highly elevated night activity. Another possibility for the birth of that myth could be in extreme projects as the ones shown in Figure 5, with a blue line showing that a peak hour of commits occurs daily very close to midnight.

In fact, a previous comparison of the work rhythms of software engineers and scientists [21] shows that scientists seem to work more during the night than software engineers. The activity of US and German scientists at night (measured by paper downloads) is roughly $42 \%$ and $25 \%$ of their day time peak activity. On the other hand, for software developers in the Mozilla and Apache projects, the corresponding percentages (based on commit count) are roughly $29 \%$ and $25 \%$ (see Fig. $1 \mathrm{~d}$ and Fig. $1 \mathrm{~b}$ ).

Of course, individuals have different working patterns and, indeed, clustering of developers revealed that one third of software developers do not seem to follow a typical office hour rhythm at all as they perform a lot of activities during evenings and weekends (see Figure 2). On the other hand, two thirds of individuals mostly follow office hours. This information is important if one wishes to build a stress detector that would use among other things commit hours, since such a detector would only work for the two thirds of software engineers who have regular working rhythms.

A manual background check of the top $10 \%$ (over 250 individuals) of Mozilla Firefox developers revealed that only half of the developers that did not follow office hours were paid by Mozilla (black line in Figure 11) as opposed to roughly $90 \%$ for the two groups that did follow office hours. This suggests that existence of hobby developers can partially explain the outside office hour activity. Still, for the middle group (blue curve in Figure 11), only $55 \%$ of commits are performed during office hours, although $90 \%$ of the individuals are paid employees, which could be a marker of overload for that group.

With respect to seniority we found no differences in working hours by using two different measures the number of commits and job title. This is rather surprising but perhaps developers that make very few commits are hobbyist and commit outside office hours while for the most senior developers the outside office hour commits would be explained by overwork.

A comparison of commit messages between office and outside office hours revealed that in our largest project, Firefox, formal code review reverts are made more often during office hours. Moreover, informal developer-initiated reverts are more often made outside office hours. Furthermore, office hour time seems to focus especially on adding new code while clean-ups are more frequent outside office hours. We found no difference in commit size, and we did not find support to the idea that as projects mature they move away from night and weekend work to more typical office hour rhythm.

In future work, we will perform a detailed qualitative analysis of both individual histories and individual developer histories to better explain the differences in working patterns, involving interviews and daily surveys to monitor project members. We will use additional data sources, such as chat logs, in order to make our set of timestamp activity more complete. Measuring the level of detachment from work, which is critical to recovery, should be studied and, similarly, normative recommendation from empirical data should be drawn. We also intend to study the impact of policies and guidelines put in place by project managers, such as a fast release cycle, on developers' activity and health. Finally, we also want to investigate further the causes of outside office hour work by focusing on periods with unusually high activity during the night or weekends.

\section{ACKNOWLEDGMENTS}

The first three authors have been supported by Academy of Finland grant 298020. The third author has been supported by Kaute foundation.

\section{REFERENCES}

[1] A. Bannai and A. Tamakoshi, "The association between long working hours and health: a systematic review of epidemiological evidence," Scandinavian journal of work, environment \& health, vol. 40, no. 1, pp. 5-18, 2014

[2] J. Greubel, A. Arlinghaus, F. Nachreiner, and D. A. Lombardi, "Higher risks when working unusual times? a cross-validation of the effects on safety, health, and work-life balance," International Archives of Occupational and Environmental Health, vol. 89, no. 8, pp. 1205-1214, Nov 2016. [Online]. Available: https://doi.org/10.1007/s00420-016-1157-z

[3] A. Wirtz, O. Giebel, C. Schomann, and F. Nachreiner, "The interference of flexible working times with the utility of time: A predictor of social impairment?" Chronobiology International, vol. 25, no. 2-3, pp. 249-261, 2008. 
[4] O. Giebel, A. Wirtz, and F. Nachreiner, "The interference of flexible working times with the circadian temperature rhythm-a predictor of impairment to health and well being?" Chronobiology International, vol. 25, no. 2-3, pp. 263-270, 2008.

[5] J. C. Hall, M. Rosbash, and M. W. Young, "The 2017 nobel prize in physiology or medicine," https://blog.sourced.tech/post/activity_hours/, 2017. [Online] Available: https://www.nobelprize.org/nobel_prizes/medicine/laureates/2017/ press.html

[6] J. W. Timothy, N. Klas, H. R. Sanghani, T. Al-Mansouri, A. T. Hughes, G. S Kirshenbaum, V. Brienza, M. D. Belle, M. R. Ralph, S. J. Clapcote et al., "Circadian disruptions in the myshkin mouse model of mania are independent of deficits in suprachiasmatic molecular clock function," Biological psychiatry, 2017.

[7] A. E. Zubidat and A. Haim, "Artificial light-at-night-a novel lifestyle risk factor for metabolic disorder and cancer morbidity," fournal of basic and clinical physiology and pharmacology, vol. 28, no. 4, pp. 295-313, 2017.

[8] C. Binnewies, S. Sonnentag, and E. J. Mojza, "Recovery during the weekend and fluctuations in weekly job performance: a week-level study examining intraindividual relationships," fournal of Occupational and Organizational Psychology, vol. 83, no. 2, pp. 419-441, 2010.

[9] S. Sonnentag, C. Binnewies, and E. J. Mojza, "Staying well and engaged when demands are high: the role of psychological detachment." fournal of Applied Psychology, vol. 95, no. 5, p. 965, 2010.

[10] A. Arlinghaus and F. Nachreiner, Unusual and Unsocial? Effects of Shift Work and Other Unusual Working Times on Social Participation. Cham: Springer International Publishing, 2016, pp. 39-57. [Online]. Available https://doi.org/10.1007/978-3-319-42286-2_3

[11] I. Spieler, S. Scheibe, C. Stamov-Roßnagel, and A. Kappas, "Help or hindrance? day-level relationships between flextime use, work-nonwork boundaries, and affective well-being." Journal of Applied Psychology, vol. 102, no. 1, pp. 67-87, 2017.

[12] R. A. Karasek Jr, "Job demands, job decision latitude, and mental strain: Implications for job redesign," Administrative science quarterly, pp. 285-308, 1979.

[13] S. Kim, E. J. W. Jr., and Y. Zhang, "Classifying software changes: Clean or buggy?" IEEE Transactions on Software Engineering, vol. 34, no. 2, pp. 181-196, March 2008.

[14] J. Eyolfson, L. Tan, and P. Lam, "Do time of day and developer experience affect commit bugginess?" in Proceedings of the 8th Working Conference on Mining Software Repositories, ser. MSR '11. New York, NY, USA: ACM, 2011, pp. 153-162. [Online]. Available: http://doi.acm.org/10.1145/1985441.1985464

[15] --, "Correlations between bugginess and time-based commit characteristics," Empirical Software Engineering, vol. 19, no. 4, pp. 1009-1039, Aug 2014. [Online] Available: https://doi.org/10.1007/s10664-013-9245-0

[16] L. Prechelt and A. Pepper, "Why software repositories are not used for defect-insertion circumstance analysis more often: A case study," Information and Software Technology, vol. 56, no. 10, pp. 1377 - 1389, 2014. [Online]. Available: http://www.sciencedirect.com/science/article/pii/S0950584914001049

[17] R. D’souza, "Why do programmers love working at night?" https://blog.sourced. tech/post/activity_hours/, 2014.

[18] S. Teller, Why programmers work at night. Leanpub, 2013.

[19] D. Robinson, "What programming languages are used late at night?" https:// stackoverflow.blog/2017/04/19/programming-languages-used-late-night/, 2017.
[20] E. A. Sall and C. R. Bhat, "An analysis of weekend work activity patterns in the san francisco bay area," Transportation, vol. 34, no. 2, pp. 161-175, 2007. [Online] Available: http://dx.doi.org/10.1007/s11116-006-0008-2

21] X. Wang, S. Xu, L. Peng, Z. Wang, C. Wang, C. Zhang, and X. Wang, "Exploring scientists' working timetable: Do scientists often work overtime?" CoRR, vol. abs/1208.2686, 2012. [Online]. Available: http://arxiv.org/abs/1208.2686

[22] M. McKee, "The weekend effect: now you see it, now you don't," BMF, vol. 353, 2016. [Online]. Available: http://www.bmj.com/content/353/bmj.i2750

[23] P. Aylin, "Making sense of the evidence for the "weekend effect"," $B M 7$, vol. 351, 2015. [Online]. Available: http://www.bmj.com/content/351/bmj.h4652

[24] B. Alexander, M. Dijst, and D. Ettema, "Working from 9 to 6 ? an analysis of in-home and out-of-home working schedules," Transportation, vol. 37, no. 3, pp. 505-523, 2010. [Online]. Available: http://dx.doi.org/10.1007/s11116-009-9257-1

[25] V. Markovtsev, "Daily commit activity on github," https://blog.sourced.tech/post/ activity hours/, 2017.

[26] F. Ferrucci, M. Harman, J. Ren, and F. Sarro, "Not going to take this anymore: Multi-objective overtime planning for software engineering projects," in Proceedings of the 2013 International Conference on Software Engineering, ser. ICSE '13. Piscataway, NJ, USA: IEEE Press, 2013, pp. 462-471. [Online]. Available: http://dl.acm.org/citation.cfm?id=2486788.2486849

[27] M. d. O. Barros and L. A. O. d. Araujo, Jr., "Learning overtime dynamics through multiobjective optimization," in Proceedings of the Genetic and Evolutionary Computation Conference 2016, ser. GECCO '16. New York, NY, USA: ACM, 2016, pp. 1061-1068. [Online]. Available: http://doi.acm.org/10.1145/2908812.2908824

[28] F. Sarro, F. Ferrucci, M. Harman, A. Manna, and J. Ren, "Adaptive multi-objective evolutionary algorithms for overtime planning in software projects," IEEE Trans. Software Eng., vol. 43, no. 10, pp. 898-917, 2017. [Online]. Available: https://doi.org/10.1109/TSE.2017.2650914

[29] F. Khomh, B. Adams, T. Dhaliwal, and Y. Zou, "Understanding the impact of rapid releases on software quality," Empirical Softw. Engg., vol. 20, no. 2, pp. 336-373, Apr. 2015. [Online]. Available: http://dx.doi.org/10.1007/s10664-014-9308-x

[30] M. Claes, M. Mäntylä, M. Kuutila, and B. Adams, "Abnormal working hours: effect of rapid releases and implications to work content," in Proceedings of the 14th International Conference on Mining Software Repositories. IEEE Press, 2017, pp. 243-247.

[31] G. Robles and J. M. Gonzalez-Barahona, "Contributor turnover in libre software projects," in IFIP International Conference on Open Source Systems. Springer, 2006, pp. 273-286.

[32] B. C. Csáji, A. Browet, V. A. Traag, J.-C. Delvenne, E. Huens, P. Van Dooren, Z. Smoreda, and V. D. Blondel, "Exploring the mobility of mobile phone users," Physica A: statistical mechanics and its applications, vol. 392, no. 6, pp. 1459-1473, 2013.

[33] Y. Hu, A. Shmygelska, D. Tran, N. Eriksson, J. Y. Tung, and D. A. Hinds, "Gwas of 89,283 individuals identifies genetic variants associated with self-reporting of being a morning person," Nature communications, vol. 7, p. 10448, 2016.

[34] J. Romano, J. D. Kromrey, J. Coraggio, and J. Skowronek, "Appropriate statistics for ordinal level data: Should we really be using t-test and cohen'sd for evaluating group differences on the nsse and other surveys," in annual meeting of the Florida Association of Institutional Research, 2006, pp. 1-33. 